\title{
Bovine Pericardium In Penile Prosthesis Reimplantation
}

\author{
Eduardo J. Lopes, Tarsila C. Santos, Modesto Jacobino \\ Section of Urology, University Hospital, Federal University of Bahia, Salvador, Bahia, Brazil
}

\begin{abstract}
We present a case of a patient who underwent a late penile prosthesis implant using bovine pericardium as a complement to the tunica albuginea involved in intense fibrosis that destroyed the corpus cavernosum after an infectious manifestation. The advantages of using bovine pericardium in the substitution of the tunica albuginea are discussed and its first use as a penile prosthesis lining is reported.
\end{abstract}

Key words: penile prosthesis; infection; graft; pericardium; cattle

Int Braz J Urol. 2007; 33: 74-6

\section{INTRODUCTION}

The incidence of infection in penile prosthesis implant surgery is approximately $3 \%(1,2)$. With this low percentage, the late implant is the most adopted option, and, eventually, the intense fibrosis that involves the corpora cavernosa makes the re-implantation of a new prosthesis more difficult $(1,2)$.

The authors report a case of a diabetic patient who presented extrusion of the prosthesis through the gland. Conservative treatment was initially adopted, followed by a late implant that required a reconstruction of the tunica albuginea with biological material.

\section{CASE REPORT}

A 62-year-old diabetic patient with a penile prosthesis implant presenting a perforation of the gland and partial extrusion of the prosthesis that had been implanted 3 years before was attended in emer- gency. As the right unit of the prosthesis perforated the gland and was partially extruded, its removal was achieved through the extrusion location. The left unit was removed through the same orifice after verification that both units were occupying the same space due to a complete destruction of the intercavernous septum. The material was collected for culture and antibiotic therapy with cephalosporin and aminoglycoside was begun. Following this, the space was cleansed with $0.9 \%$ physiological saline containing gentamicin. A suture of the corpus cavernosum was performed using vycril 2-0 and the orifice of the glandar corpus spongiosum was sutured with chromecatgut 3-0 in 2 simple stitches. The result of the culture revealed the presence of Staphylococcus aureus and Streptococus epidermidis sensitive to the adopted antibiotic therapy.

Sixty days after the resolution of the infectious condition, a new AMS 650 prosthesis was reimplanted through penoscrotal access. The prophylactic antibiotics used were cefazolin ( $1 \mathrm{~g} / \mathrm{IM} /$ day for 72 h), gentamicin sulfate $(80 \mathrm{mg} / \mathrm{IM}, 8$ every 8 hours 
for $48 \mathrm{~h}$ ), metronidazol ( $400 \mathrm{mg}$ IV of 8 every $8 \mathrm{~h}$ for $48 \mathrm{~h}$ ), and gentamicin sulfate (160 $\mathrm{mg}$ in physiological saline solution) where the prosthesis was submersed. Due to the intense fibrosis and the small space within the tunica albuginea, it was only possible to implant a single unit. We opted for the inverted insertion of the prosthesis in the distal extremity of the tunica albuginea using the small space inside the corpus cavernosum (Figures-1 and 2). The $4 \mathrm{~mm}$ bovine pericardium was used to line the entire extension of the implanted prosthesis, complementing the fibrosed tunica albuginea (Figure-3). Two years later, the patient is satisfied with the result and has been maintaining regular sexual relations without any complaints or recurrences.

\section{COMMENTS}

When infection occurs in a penile prosthesis implant, 2 procedures can be adopted: 1) a rescue procedure, which requires an exhaustive cleansing of the area, antibiotic therapy and the re-implantation of a new prosthesis at the same time (this is considered an exception despite the good result obtained by some authors (2); and 2) late implant, necessitating removal of the prosthesis, local cleansing, specific treatment for the infection and implant of a new prosthesis following remission of the infection (2).

We chose the second option because of our greater experience with it and because this procedure is more discussed and accepted. The period between the removal of the prosthesis and the re-implant remains uncertain, varying from 2 to 39 months (with a mean of 8 months) $(1,2)$. In this case, we opted for the implant of a new prosthesis 2 months after the first intervention.

Even though synthetic materials are available and inactive, they have the disadvantage of presenting a larger rate of infection, inelasticity and fibrosis of the corpora cavernosa $(1,2)$. On the other hand, the tensional strength of the biological material has been questioned, this material is not readily available and has a larger risk of diseases transmission (1).

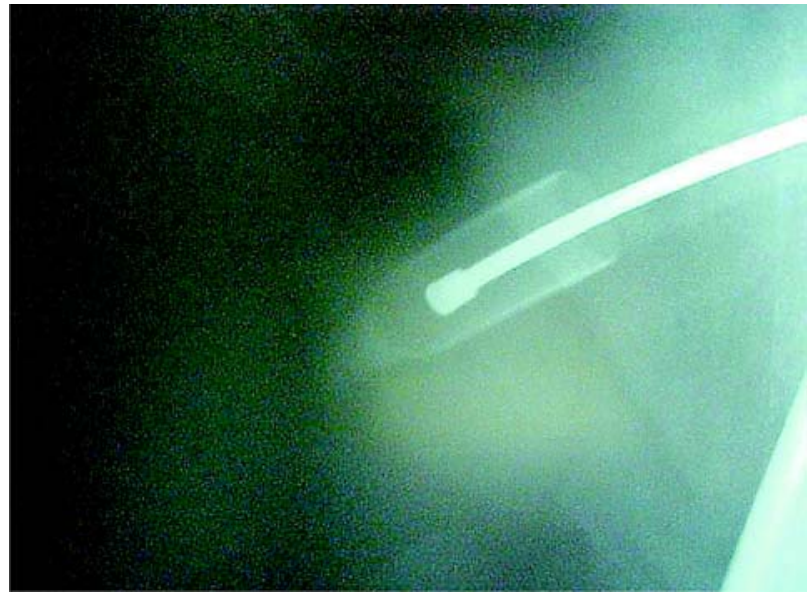

Figure $1-R x$ showing a single prosthesis implanted in an inverted fashion.

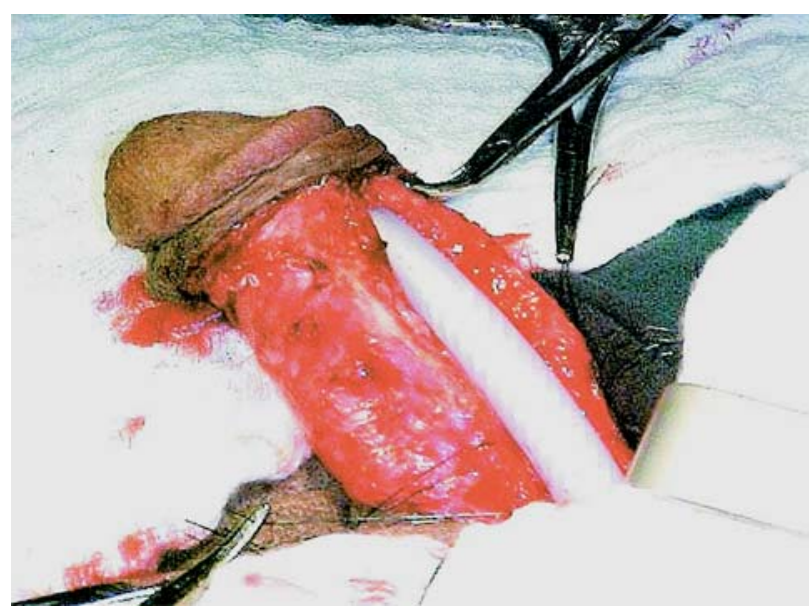

Figure 2-AMS 650 prosthesis with the inferior extremity inserted in the gland.

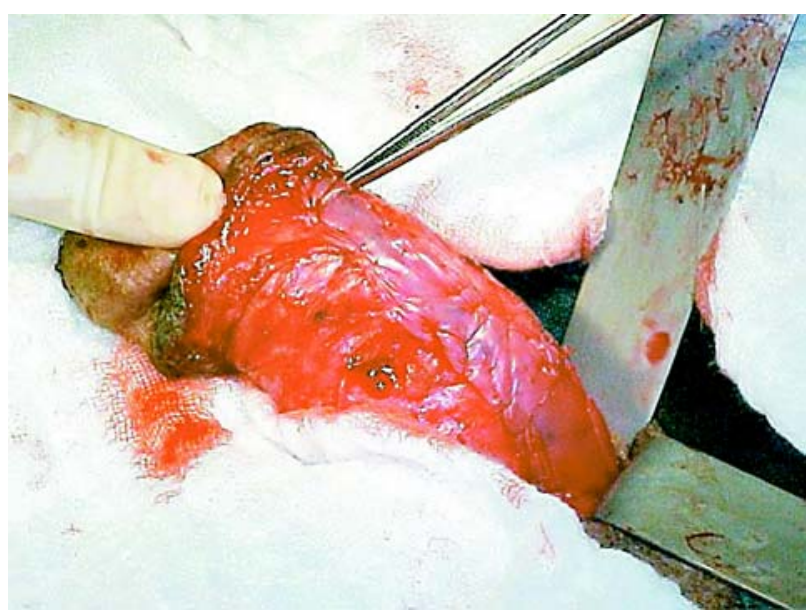

Figure 3 - Aspect of the prosthesis lined with the bovine pericardium. 
We have chosen bovine pericardium because of its lower cost, inertia, elasticity, absence of transmission of diseases, low probability of retraction and good resistance to tension that allow it to cover large defects without forming protuberances (3). Despite having never been used as a lining for a penile prosthesis, we believe that it is another option in extreme situations such as the one we are describing.

\section{CONFLICT OF INTEREST}

None declared.

\section{REFERENCES}

1. Palese MA, Burnett AL: Corporoplasty using pericardium allograft (tutoplasty) with complex penile prosthesis surgery. Urology. 2001; 58: 1049-52.

2. Mulcahy JJ, Brant MD, Ludlow JK: Management of infected penile implants. Tech Urol. 1995; 1: 115-9.

3. Pomerantzeff PM, Zerbini EJ, Verginelli G, Jatene AD: Valve replacement in the Heart Institute, University of Sao Paulo, Brazil. Ann Thorac Surg. 1989; 48: S414.

Accepted after revision:

July 29, 2006

\author{
Correspondence address: \\ Dr. Eduardo José Andrade Lopes \\ Rua Altino Serberto de Barros, 241 / 405 \\ Ed. Memorial Itaigara \\ Salvador, BA, 41850-010, Brazil \\ Fax: + $55713351-7424$ \\ E-mail: ejalopes@terra.com.br
}

